\title{
A NEW APPROACH FOR 3D SEGMENTATION OF CELLULAR TOMOGRAMS OBTAINED USING THREE-DIMENSIONAL ELECTRON MICROSCOPY
}

\author{
A. Bartesaghi and G. Sapiro* \\ University of Minnesota \\ Electrical and Computer Engineering Department \\ Minneapolis, MN 55455
}

\author{
S. Lee, J. Lefman and S. Subramaniam ${ }^{\dagger}$ \\ National Institutes of Health \\ National Cancer Institute \\ Bethesda, Maryland 20892
}

\begin{abstract}
Electron tomography allows determination of the threedimensional structures of cells and tissues at resolutions significantly higher than is possible with optical microscopy. Electron tomograms contain, in principle, vast amounts of information on the locations and architectures of large numbers of subcellular assemblies and organelles. The development of reliable quantitative approaches for interpretation of features in tomograms, is an important problem, but is a challenging prospect because of the low signal-to-noise ratios that are inherent to biological electron microscopic images. As a first step in this direction, we report methods for the automated statistical analysis of HIV particles and selected cellular compartments in electron tomograms recorded from fixed, plastic-embedded sections derived from HIV-infected human macrophages. Individual features in the tomogram are segmented using a novel, robust algorithm that finds their boundaries as global minimal surfaces in a metric space defined by image features. Our expectation is that such methods will provide tools for semi-automated detection and statistical evaluation of HIV particles at different stages of assembly in the cells, and present opportunities for correlation with biochemical markers of HIV infection.
\end{abstract}

\section{INTRODUCTION}

Transmission electron microscopes have conventionally been used in biomedical research to obtain two-dimensional projection images of thin objects such as molecules, cells and tissues. Such images can be recorded in most modern electron microscopes at magnifications ranging from $\sim 100 \mathrm{x}$ to $\sim 1,000,000 x$. The use of electron microscopes, is, however, not limited to imaging in 2D. Using emerging methods in electron tomography (see [1] for a recent review), it is now also possible to routinely determine three-dimensional structures using principles that are very similar to those used

\footnotetext{
${ }^{*}$ Work supported by NSF, ONR, and NIH. Authors are also with the Digital Technology Center.

${ }^{\dagger}$ Work supported by NIH.
}

in technologies such as computerized axial tomography. Thus, one can record a series of images of a given object over a wide range of tilt angles, and combine them using back projection algorithms to generate three-dimensional volume of the imaged object.

A key problem in biological electron tomography is that the images obtained are at relatively low signal-to-noise ratios. In part, this is because of the tremendous complexity of biological specimens; for example a single human cell can contain thousands of copies of tens of thousands of proteins packaged in a variety of multi-protein complexes and organelles of differing shapes and sizes. A second factor comes from the potential of electrons to damage organic matter, which necessitates the use of electron doses that are high enough to obtain measurable contrast, but low enough to minimize structural damage. The rapid, quantitative interpretation of the vast amount of data in tomograms of cells and tissues therefore poses a challenging problem. This issue is becoming increasingly important now because of the rapid advances in instrument automation that have led to dramatic enhancements in the speed of data collection. We are interested in developing approaches for 3D segmentation of features in cellular tomograms that can work robustly and rapidly despite the low signal-to-noise ratios. As a test case, we have used tomograms recorded from human macrophages infected with HIV. The cells were fixed, embedded in plastic, stained with uranyl acetate and lead citrate and sectioned in an ultramicrotome to produce sections with thicknesses in the range of $150 \mathrm{~nm}$ to $200 \mathrm{~nm}$. These sections were placed on an electron microscopic grid coated with a thin carbon film, and imaged in a Tecnai 12 electron microscope operating at $120 \mathrm{kV}$ equipped with $\mathrm{a} \mathrm{LaB}_{6}$ filament. Tomograms were constructed using standard backprojection algorithms as implemented in the IMOD reconstruction package [2].

Figure 1 shows slices from a tomogram recorded from a small region of cells infected with HIV. Within this slice, there are several identifiable features which bear a resemblance to the slice of either an assembled virion or enclosed membranous entities with varying interior density relative 
to the cytoplasmic medium. Our goal is to detect these structures with minimal user bias, analyze them, and establish correlation of the nature and extent of these features with progression of viral infection. In this work, we concentrate primarily on the segmentation of these features and basic statistical analysis of their distribution in the volume.
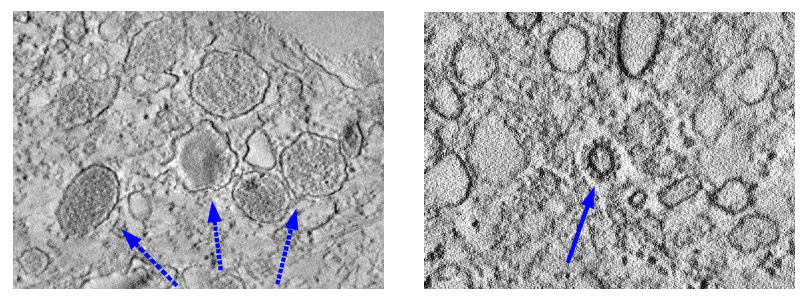

Fig. 1. Four types of features identified manually in a slice extracted from a tomogram. The tomogram was obtained from a chemically fixed, plastic-embedded, stained $200 \mathrm{~nm}$ thick section from an HIV-infected macrophage. Each of them has an envelope that is stained more darkly than the background. Some such as the one pointed to by the solid arrow resemble an assembled HIV particle, while others, such as those pointed to by the dashed arrows are vesicular in nature and could represent entities that are either precursors or packaging units for the fully assembled virus.

\section{3D TOMOGRAPH SEGMENTATION}

As pointed out above, electron tomographic images generally display very low signal-to-noise ratios, and the task of segmenting features of interest can be a challenging one. We are developing new algorithms that can work under these conditions, as described below.

As all the features of interest have a dark boundary around them (i.e. only the inside is different), we will use the same segmentation algorithm to get the boundary, and at the second stage, classify them by looking at the interior voxels. Based on very simple criteria (i.e. average gray value) we can separate filled from empty structures, and more complex criteria can be used to achieve a finer classification (see Section 4).

The tomograms are segmented in a semi-automatic fashion: a single point inside each cell is first specified by the user selecting only those structures that correspond to simple closed boundaries (this is done by inspection of all slices in the $3 \mathrm{D}$ volume). For each selected point we segment the surrounding structure using a robust segmentation algorithm described next. Although the detection of interior points can be done automatically (e.g., from singularities of the distance function to robust edges), the structure of the tomograms is so complex (membranes merged together, boundaries with large gaps, presence of secondary structural elements, etc.), that it will require intensive post-processing to eliminate irrelevant objects that are not meaningful for the subsequent analysis. We should also note that at the present stage of the investigation, our goal is to select as many features as possible in order to minimize user bias in particle selection.

\subsection{Segmentation of individual features}

The segmentation problem is formulated as the search for a minimal surface on a metric space that depends on image features, with the restriction that it contains the specified interior point. Let's $\mathcal{S}$ denote the 3D surface representing the boundary. The minimal surface problem is stated as [3]:

$$
\mathcal{S}=\underset{\mathcal{S}_{p}}{\arg \min } \int_{\mathcal{S}_{p}} g\left(\mathcal{S}_{p}\right) d s
$$

where the metric $g: \Re^{3} \rightarrow[0, \infty]$ will signal whether or not a point is in the boundary of a feature element. Specifically, $g$ will take low values for points located over thin dark regions and higher values elsewhere in the volume. The minimal surface $\mathcal{S}$ is then encouraged to go through areas of small $g$, avoiding regions of high $g$. As in every energy minimization setting, two issues have to be addressed: first, find the appropriate $g$ metric so the surface will follow the features of interest; and second, solve the minimization problem to get the globally optimal surface.

\subsubsection{Designing the image dependent metric}

The goal is to define a metric that captures the local geometry of the image and signals the presence of flat dark areas which correspond to 3D boundary voxels. Following the work in [4] we look at eigenvectors and eigenvalues of the Hessian matrix to characterize the local structure of an image. Let's $\lambda_{k}$ denote the eigenvalue with the $k$-th smallest magnitude (i.e. $\left|\lambda_{1}\right| \leq\left|\lambda_{2}\right| \leq\left|\lambda_{3}\right|$ ). Plate-like structures have one predominant eigenvalue and corresponding eigenvector in the direction normal to the plane (the direction where gray value changes the most). The other two eigenvalues have vanishing values and the corresponding eigenvectors form an orthogonal basis of the plane. By looking at the sign of the first eigenvalue, we can tell apart dark platelike features $\left(\lambda_{1}>0\right)$ from bright ones $\left(\lambda_{1}<0\right)$. Combining these factors together we define a geometric measure function $\mathcal{M}: \Re^{3} \rightarrow[0, \infty]$ as:

$$
\mathcal{M}=\left\{\begin{array}{cc}
0 & \lambda_{1}>0 \\
\sqrt{\lambda_{1}^{2}+\lambda_{2}^{2}+\lambda_{3}^{2}} \cdot\left|\lambda_{1}\right| & \text { otherwise }
\end{array}\right.
$$

In a post-processing step, a non-maxima suppression algorithm keeps only local maxima of $\mathcal{M}$ in the direction perpendicular to the plane (this is the 3D extension of the technique used in the Canny edge detector, see [5]). By keeping 
only local maxima in the direction normal to the plane we obtain a better localized feature indication function.

Observe that the bigger the value of $\mathcal{M}$ at a given point, the stronger the indication that the point may be on the boundary of a structure. We then choose the metric $g$ to be $g(\mathcal{M})=\left(1-\mathcal{M}_{n}\right)+w$, where $\mathcal{M}_{n}=\frac{\mathcal{M}}{\max (\mathcal{M})}$ is the normalized measure $\left(0 \leq \mathcal{M}_{n} \leq 1\right)$, and $w$ is a small constant that prevents $g$ from vanishing, see [6]. As required, $g \rightarrow 1+w$ for background voxels $\left(\mathcal{M}_{n} \rightarrow 0\right)$ and $g \rightarrow w$ for points located on the boundaries of interest $\left(\mathcal{M}_{n} \rightarrow 1\right)$.

\subsubsection{Finding the minimal surface}

Once the metric is defined, we are ready to solve the minimization problem and get the surface $\mathcal{S}$ that minimizes the energy (1). This problem is usually solved in a variational framework, we compute the Euler-Lagrange equations and find the optimal way to reduce the energy (1) given an initial energy state. Unfortunately, this will only find a local minimizer (the closest one to the initial condition) and cannot guarantee the convergence to the global minima. Many algorithms have been proposed in the literature that provide different mechanisms trying to drive the surface towards the global minima, e.g. [7, 8]. Although they offer improved performance, they can still get trapped in local minima. There are a few approaches that guarantee convergence to the global minima in the 2D case (curves on 2D images), see [6,9]. For the 3D case, the recent work [10] finds the global minima for surfaces but requires a pair of curves that lay on the object as initialization.

Our approach for finding the minimal surface is inspired by [9], extended to surfaces instead of planar curves. The only input required by the algorithm is a point inside the cell. The surface minimization problem is then solved imposing the restriction that the initial point should be inside the surface. A 3D polar coordinate transformation is first performed with center in the point inside the cell. We then look for an initial surface guess in polar space, using a technique similar to the one in [11]. The resulting surface (that we express in implicit representation) is back-converted from polar coordinates to the Cartesian grid along with the intrinsic distances (computed in the polar domain with the $g$ metric). Back on the Cartesian grid, we denote $\phi$ the implicit surface representation and $d$ the back converted distances. As a refinement step, $\phi$ is evolved according to the following partial differential equation: $\phi_{t}=\kappa|\nabla \phi|+$ $\mathcal{M}_{n}(\nabla d \cdot \nabla \phi)$, where $\mathcal{M}_{n}$ is the normalized feature indicator function defined before. The curvature term is a regularity constraint and the external advection field $\nabla d$ pushes the surface towards the minima of the energy (1). The factor $\mathcal{M}_{n}$ turns the advection term off in voxels that are not on the boundary $\left(\mathcal{M}_{n} \rightarrow 0\right)$, so pure regularization will nicely fill-in the missing gaps.
The segmentation for each 3D structure is run independently and in a serial fashion. The approximate running time for each of them is less than 3 minutes in a $1.2 \mathrm{Mhz}$ laptop computer. The processing of the whole volume can well be parallelized to significantly reduce the computation time. Some examples are shown in the next section.

\section{RESULTS}

In Figure 2 we show representative resulting 3D surfaces superposed on top of slices of an unprocessed tomogram. The segmented surface shows an excellent fit to the boundaries of the vesicular features. We also show that the segmentation algorithm can be used to classify the volumes in terms of the mean internal density, as illustrated in a segmented 2D slice, where the regions are automatically classified (red and green) based on differing internal average grey values. We also show a 2D slice of the full tomogram with the segmentation curves superposed, Figure 3.
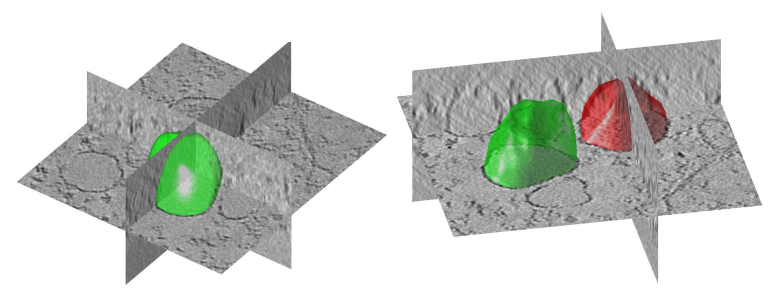

Fig. 2. Two examples of 3D segmentation of cell structure generated from a point inside specified by the user. The right example shows volumes corresponding to low density (green) and to high density (red).

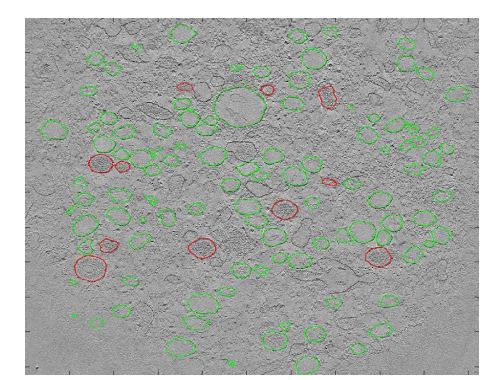

Fig. 3. We show a slice of the $3 \mathrm{D}$ volume with the segmentation curves on top. Two density classes are shown (red and green) automatically classified according to the average gray level inside the volumes.

Once all relevant structures are segmented, we can perform some simple statistical analysis on the results. As each volume is obtained as an implicit function, geometry computations (e.g. size, average gray value, shape, etc.) are easily obtained. In Figure 4 we show histograms of the average 
gray level (density) distributions inside the selected volumes in two different tomograms. Note that we can clearly classify cells into two classes: the ones with the filled interior and the empty ones. Furthermore, looking at the spatial distribution of gray values inside each cell, more sophisticated criteria can be devised to classify in the different cell types presented in Section 1.
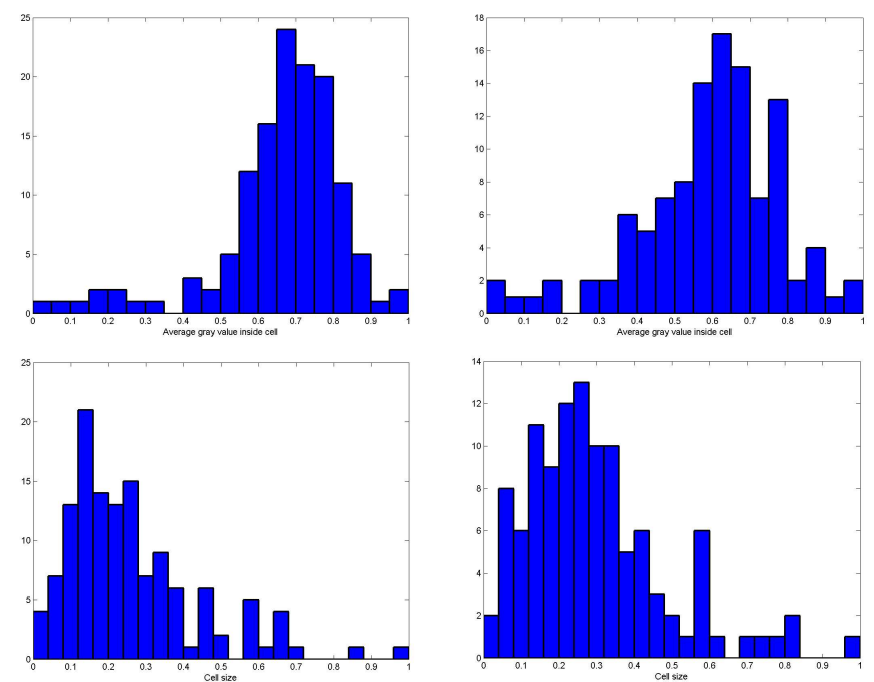

Fig. 4. Top: Distribution of average gray levels inside segmented regions in two different tomograms from different regions of the infected cell. Bottom: Distribution of size in segmented volumes within a tomogram, normalized with reference to the largest volume in the set.

Figure 4 also shows the size distribution of cells within each tomogram. Note that as overall thickness of the tomogram is only $\sim 200 \mathrm{~nm}$, and the virion/vesicular entities are $\sim 100 \mathrm{~nm}$ wide, only a few cells are captured completely inside the volume. Acquisition of serial tomograms from successive sections that are stitched together computationally should allow analysis of larger volumes, therefore providing more reliable statistics on the segmented volumes.

\section{CONCLUSION}

In this paper we have report a new algorithm specifically designed for the semi-automated segmentation of features in cellular tomograms obtained by electron microscopy. We demonstrate this is applicable for the identification and possible classification of HIV particles in infected macrophages, and could provide a quantitative basis for analyzing HIV infection.

We are currently acquiring new data to study the evolution of the statistics (see for example Figure 4) when the virus evolves. In addition to simple statistics such as average gray value (representing the density inside the region) and volume, we plan to carry out shape analysis and classification of the segmented regions using novel computational approaches for shape statistics being developed in the computer vision literature.

\section{REFERENCES}

[1] S. Subramaniam and J. L. S. Milne, "Threedimensional electron microscopy at molecular resolution," Ann. Rev. Biophys. and Biomol. Struct., vol. 33, June 2004, in press.

[2] J. R. Kremer, D. N. Mastronarde, and J. R. McIntosh, "Computer visualization of three-dimensional image data using IMOD," Journal Of Structural Biology, , no. 116 , pp. 71-76, 1996.

[3] G. Sapiro, Geometric Partial Differential Equations and Image Processing, Cambridge University Press, New York, 2001.

[4] A. F. Frangi, W. J. Niessen, K. L. Vincken, and M. A. Viergever, "Multiscale vessel enhancement filtering," Lecture Notes in Computer Science, vol. 1496, 1998.

[5] Q. Lin, Enhancement, Extraction, and Visualization of 3 D Volume Data, Dissertations no. 824, isbn 91-7373657-0, Linkping University, Sweden, 2002.

[6] L. D. Cohen and R. Kimmel, "Global minimum for active contour models: A minimal path approach," IJCV, vol. 24, no. 1, pp. 57-78, August 1997.

[7] L. D. Cohen, "On active contour models and balloons," Computer Vision, Graphics and Image Processing., vol. 53, no. 2, pp. 211-218, 1991.

[8] C. Xu and J.L. Prince, "Gradient vector flow: A new external force for snakes," Proc. IEEE Conf. on Comp. Vis. Patt. Recog. (CVPR), vol. Los Alamitos: Comp. Soc. Press, pp. 66-71, June 1997.

[9] B. Appleton and H. Talbot, "Globally optimal geodesic active contours," Journal of Mathematical Imaging and Vision, submitted, 2002.

[10] R. Ardon and L. D. Cohen, "Fast constrained surface extraction by minimal paths," in 2nd IEEE Workshop on Variational, Geometric and Level Set Methods in Computer Vision, Olivier Faugeras and Nikos Paragios, Eds., Acropolis, Nice, France, October 2003, pp. 233-240.

[11] C. Sun, "Fast stereo matching using rectangular subregioning and 3D maximum-surface techniques," International Journal on Computer Vision, vol. 47, no. 1/2/3, pp. 99-117, May 2002. 